\title{
Zielgerichtete RCC-Therapie mit Sunitinib
}

— Die aktuellen Leitlinien der European Association of Urology empfehlen für die Erstlinientherapie des fortgeschrittenen Nierenzellkarzinoms (RCC) bei niedrigem bis mittlerem Risiko Sunitinib, bei hohem Risiko Temsirolimus. Diese Empfehlung wird in Deutschland in Klinik und Praxis weitgehend umgesetzt. Laut Tumorregister werden etwa zwei Drittel aller RCC-Patienten mit dem Multikinase-Inhibitor Sunitinib (Sutent ${ }^{\circledR}$ ) behandelt, an zweiter Stelle folgt mit etwa 13\% der mTOR-Inhibitor Temsirolimus (Torisel ${ }^{\circledR}$ ). Das Register bestätigt die Daten der PhaseIII- und IV-Studien hinsichtlich der Effektivität mit hohen Ansprechraten, PFS-Verdopplung (PFS = progressionsfreies Überleben) und Lebensverlängerung sowie hinsichtlich der
Nebenwirkungen, die selten Grad 3/4 erreichen.

Die TWiST-Analyse (Time Without Disease Progression or Toxicity) der Zulassungsstudien zeigte nun, dass die verlängerte Lebenszeit - 6,4 Monate für Sunitinib, 3,6 Monate für Temsirolimus, jeweils verglichen mit Interferon (IFN) - nicht mit einem Verlust an Lebensqualität einhergeht [Patil S et al. J Clin Oncol 2010; 28: 7s]. Unter den aktuell verfügbaren zielgerichteten Therapieoptionen existierten nur für die beiden genannten Substanzen entsprechende Lebensqualitätsdaten, erklärte Prof. Thomas Otto, Neuss. Die Analyse weist nach, dass die Zeit ohne Symptome und Toxizität unter Sunitinib fast doppelt so lang ist wie unter IFN (335 Tage versus 172 Tage), bei lediglich 19 Tagen, die mit Grad 3/4-Nebenwirkungen assoziiert waren. Ähnlich die Daten zu Temsirolimus: Die Zeit ohne Symptome und Toxizität ist unter der mTOR-Inhibition um 38\% verlängert gegenüber der IFN-Therapie.

"Länger und besser leben, ohne klinische Symptome und ohne durch die Erkrankung belästigt zu werden - das sind für den Patienten die entscheidenden Therapieziele", sagte Otto.

koc

Pressekonferenz „Behandlungsrealität metastasiertes Nierenzellkarzinom (mRCC) Update 2010", Berlin 19. November 2010 Veranstalter: Pfizer Oncology, Berlin

\section{Infos rund um die Belastungsinkontinenz per Mausklick}

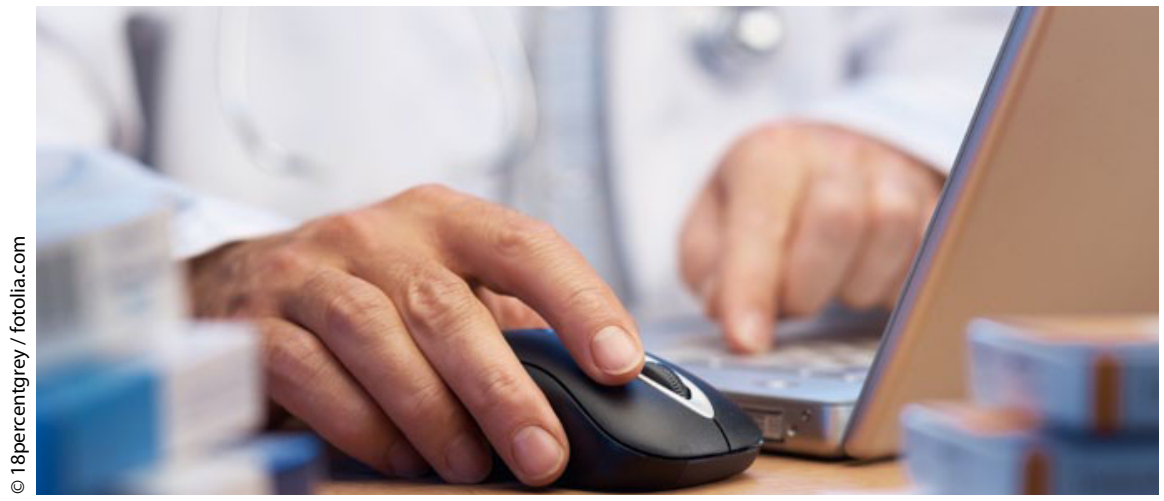

Ärzte können sich auf der Yentreve-Website über die Therapie mit Duloxetin informieren.

_ Der kürzlich neu gestaltete InternetAuftritt www.yentreve.de bietet nicht nur für Patienten wissenswerte Informationen, auch Mediziner kommen hier auf ihre Kosten. Ärzte finden beispielsweise dank der einfachen Navigationsführung innerhalb kurzer Zeit Antworten auf ihre Fragen zur Therapie der weiblichen Belastungsinkontinenz. In einem Literaturarchiv stehen Studien und Hintergrundartikel zur Wirksamkeit und Verlässlichkeit der Behandlung mit Duloxetin (Yentreve ${ }^{\oplus}$ ) zur Verfügung. Alles Wissenswerte rund um Yentreve ${ }^{\circledR}$ ist in der Rubrik "Auf einen Blick" in kurzer Form zusammengefasst. Zusätzlich werden häufige Fragen wie "Wann ist ein Wirkungseintritt zu erwarten?" oder "Wie lange kann Yentreve ${ }^{\circledR}$ einge- nommen werden?" kurz und knapp beantwortet. Dadurch sind die wichtigsten Informationen auch während der Behandlung schnell zur Hand. Ärzte und medizinisches Fachpersonal erhalten auf der YentreveWebsite zudem die Möglichkeit, per OnlineFortbildung ihr Wissen zur Harn- und vor allem zur Belastungsinkontinenz vertiefen. Für Patienten hat die Website auch einiges zu bieten: So steht beispielsweise ein Miktionstagebuch, das direkt ausgedruckt werden kann, bereit. Zusätzliche Informationsmaterialien wie Beckenbodenbroschüren oder Anamnese-Fragebögen sind ebenfalls erhältlich.

Nach Informationen von Lilly, Bad Homburg

\section{Neuer Männerkatheter wahrt die Diskretion}

_ Seit Januar 2011 ist ein neuer, sofort gebrauchsfertiger Einmalkatheter für Männer auf dem Markt, der aufgrund seines diskreten Designs nicht auf den ersten Blick als solcher erkennbar ist: der SpeediCath ${ }^{\circledR}$ Compact für Männer. Der Katheter lässt sich von $19 \mathrm{~cm}$ Verpackungsgröße nach dem Teleskopprinzip auf eine Gesamtlänge von $30 \mathrm{~cm}$ ausziehen. Dabei hat der flexible vordere Teil des Katheters eine Länge von $17,5 \mathrm{~cm}$, der hintere steifere Teil eine Länge von $12,5 \mathrm{~cm}$. Der Durchmesser vergrößert sich in einem fließenden Übergang von $\mathrm{CH} 12$ auf $\mathrm{CH} 18$. Die Handhabung des Katheters ist einfach: Anwender brauchen die Katheterhülle nur aufzudrehen und die ineinandergesteckten Röhrchen auseinanderzuziehen. Der steife Teil des Katheters erleichtert das gezielte Einführen in den Harnröhreneingang. Der PVC- und phthalatfreie Einmalkatheter kann an jeden Standard-Urinbeutel angeschlossen werden. Nach Gebrauch lässt sich der Katheter in die Verpackung zurückschieben und somit diskret entsorgen.

Nach Informationen von

Coloplast, Hamburg 